\title{
Transition from longitudinal to transversal patterns in an anisotropic system
}

\author{
Martin May, ${ }^{*}$ Wolfgang Schöpf, ${ }^{\dagger}$ and Ingo Rehberg ${ }^{*}$ \\ Experimentalphysik V, Universität Bayreuth, D-95440 Bayreuth, Germany \\ Alexei Krekhov ${ }^{\S}$ \\ Theoretische Physik I, Universität Bayreuth, D-95440 Bayreuth, Germany
}

Agnes Bukall

Research Institute for Solid State Physics and Optics of the Hungarian Academy of Sciences, H-1525 Budapest, Hungary

(Received 25 June 2008; revised manuscript received 25 August 2008; published 24 October 2008)

\begin{abstract}
Periodic stripe patterns which form when an electric field is applied to a thin nematic liquid crystal layer with a very low conductivity are discussed. In this case the dielectric electroconvection mode persists down to very low frequencies of the driving voltage. A Lifschitz point, i.e., a transition from normal to oblique rolls is detected in the dielectric regime. A crossover from electroconvection to flexoelectric domains occurs for extremely low frequencies of about $0.1 \mathrm{~Hz}$. The crossover scenario yields pattern morphologies characteristic for both mechanisms, i.e., electroconvection and flexoelectric domains which appear consecutively within one period of the driving voltage. A theoretical description of the onset characteristics of dielectric convection, which is based on an extended model including flexoelectricity, is also presented.
\end{abstract}

DOI: 10.1103/PhysRevE.78.046215

PACS number(s): 47.54. $-\mathrm{r}, 61.30 .-\mathrm{v}, 47.65 .-\mathrm{d}$

\section{INTRODUCTION}

Pattern formation in isotropic and in anisotropic systems is fundamentally different. If an isotropic system undergoes a pattern-forming instability, the wave vector of the resulting periodic pattern may have any orientation within this system (see, e.g., Ref. [1]). A classic example is the RayleighBénard convection of an isotropic fluid in a rotationally symmetric setup [1-3]. If the system has a preferred direction and is thus anisotropic, either through intrinsic mechanisms or by external forcing, the typical orientation of the arising pattern is parallel or perpendicular to this direction. In Rayleigh-Bénard convection in a narrow channel, e.g., the rolls align perpendicular to the sidewalls with the wave vector in the preferred direction, i.e., along the channel. If, on the other hand, Rayleigh-Bénard convection is considered in a parallel flow field, such as plane Pouiseuille or Couette flow, the rolls tend to align with their axis parallel to the flow direction, i.e., with the wave vector perpendicular to the preferred direction [4,5]. Thus, a competition between the two orientations may be observed in special geometries. Another example is the solidification pattern formed on the propagating interface of a supercooled liquid, where dendrites grow in the direction from solid to liquid. Depending on the symmetry of the crystalline phase, side branches develop perpendicularly in the case of a four-fold symmetry, under $60^{\circ}$ for a hexagonal symmetry, and so on $[1,6]$.

Prime examples for pattern forming phenomena in anisotropic systems are the electric field induced structures in

\footnotetext{
*martin.may@uni-bayreuth.de

†wolfgang.schoepf@uni-bayreuth.de

†ingo.rehberg@uni-bayreuth.de

§alexei.krekhov@uni-bayreuth.de

"ab@szfki.hu
}

nematic liquid crystals [7-9]. Here, both orientations as well as intermediate, namely oblique roll directions can be observed for the resulting patterns, depending on the material parameters and the experimental conditions $[10,11]$. An example for a transition from parallel to perpendicular orientation is shown in Fig. 1, with the details being explained later. In this paper, we focus our interest on electroconvection [Figs. 1(b) and 1(c)] and on flexoelectric domains [Fig. 1(a)] as well as on the transition between the two.

Most nematic liquid crystals have a uniaxial orientational order which is represented by the unit vector $\hat{n}$, the director $[12,13]$. Electroconvection has most commonly been observed in planarly oriented layers, where the liquid crystal is placed between two transparent electrodes which have been treated in such a way, that the director is fixed in the plane of the electrodes and thus forced into a preferred direction. If a sinusoidal ac voltage is applied across the electrodes, then in case of an appropriate combination of material parameters electroconvection manifests itself above a threshold voltage as a system of parallel convection rolls. The arising patterns have great morphological richness already at onset: Normal rolls, where the wave vector is parallel to the initial director orientation, oblique rolls, where the wave vector encloses a finite angle with this direction, as well as stationary and traveling rolls have been detected, depending on the driving frequency (for an overview, see, e.g., Refs. $[1,14]$ ). The patterns are excited by the Carr-Helfrich mechanism, which leads for a sufficiently large voltage to a positive feedback so that specific director fluctuations can grow and a periodic director modulation develops $[15,16]$. Because the director represents the optical axis of the liquid crystal, this leads to a modulation of the refractive indices. Thus, the pattern becomes optically detectable as dark and bright stripes by using the shadowgraph technique [17] (see also Fig. 1).

These scenarios have been described theoretically by the so-called standard model of electroconvection, incorporating the coupling between the charge, velocity, and director fields 

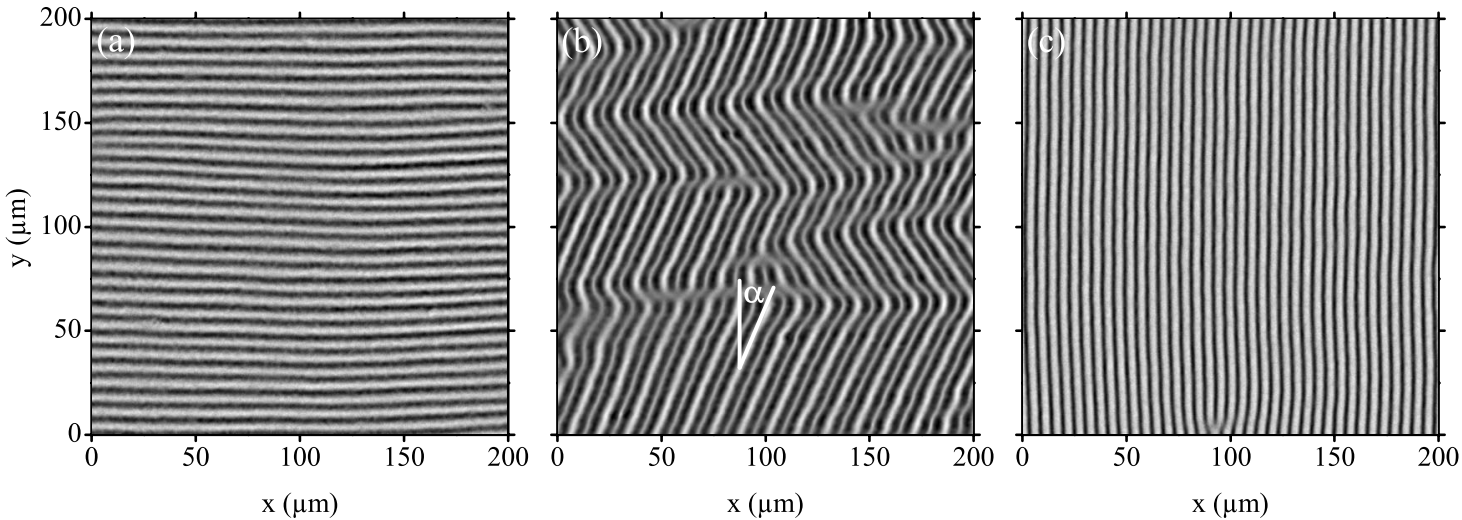

FIG. 1. Snapshots of the various patterns as seen from above with light linearly polarized along the $x$ direction. (a) Flexoelectric domains at a dc voltage of $U=14.39 \mathrm{~V}$. (b) Oblique dielectric convection rolls at $f=4 \mathrm{~Hz}$ and $U=13.05 \mathrm{~V}$. $\alpha$ is the angle of obliqueness. (c) Normal dielectric convection rolls at $f=150 \mathrm{~Hz}$ and $U=48.01 \mathrm{~V}$.

of the liquid crystal $[7,8,18,19]$. Various predictions of this model have been compared with experimental results (see, e.g., Refs. $[8,20,21])$. Depending on the frequency $f$ of the driving ac voltage, two different convection regimes exist. Below the crossover frequency $f_{c}$, in the conductive regime, the charge distribution oscillates with $f$, while the director and velocity fields are stationary in leading order. Above $f_{c}$, in the dielectric regime, the situation is reversed: The charge distribution is stationary in leading order while the director and the velocity fields oscillate with $f$. The two regimes have different characteristics with respect to the dependence of the critical voltage on the frequency and on the thickness. The crossover frequency $f_{c}$ between the two modes depends significantly on the conductivity of the liquid crystal used, as well as on the sample thickness $d$. In order to extend the frequency range of the dielectric regime downwards, i.e., to shift $f_{c}$ to lower values, one must reduce either the cell thickness or the electric conductivity or both. When both are sufficiently small, the conductive regime may not occur at all.

Nematic liquid crystals are typically regarded as regular dielectrics, where the source of the electric polarization is the electric field. However, the flexoelectric polarization $P_{f}$ may appear even in the absence of an electric field, if the director field is distorted $[12,13,22]$. The magnitude of $P_{f}$ is determined by two material parameters, the flexoelectric coefficients $e_{1}$ and $e_{3}$. Flexoelectricity may influence the electroconvection scenario by producing an additional contribution to the charge distribution and to the electric torque acting on the director. It is also known, that under a sufficiently large dc voltage, flexoelectricity may distort the director field in the planar geometry, resulting in a nonconvective pattern of stripes parallel to the preferred direction [see Fig. 1(a)]. These stripes are called flexoelectric domains or flexodomains $[23,24]$. A qualitative explanation of these domains has been given in the framework of the electrostatic equations of nematic liquid crystals, and threshold formulas for the voltage and wavelength have been obtained in a oneconstant approximation. The frequency dependence, however, has not been studied at that time [23,24].

The influence of flexoelectricity on electroconvection has been studied theoretically $[19,25-27]$ and it has been shown that it establishes a coupling between the conductive and dielectric modes resulting in a complex time dependence $[27,28]$. It has been shown earlier that flexoelectricity decreases the dc threshold voltage of electroconvection by about $25 \%$ (for the material parameters of the nematic liquid crystal MBBA) but leaves the ac threshold of the conductive mode unaltered, at least for not too thin cells and sufficiently high frequencies [19]. The limit of $f \rightarrow 0 \mathrm{~Hz}$, i.e., the character of the transition from ac to dc driving has not been studied rigorously so far, neither experimentally nor theoretically.

In this paper we report experimental investigations of electroconvection when the dielectric mode extends to very low frequencies (no conductive regime is observed in our sample) and compare our results with numerical simulations which are based on an extended standard model including flexoelectricity $[27,28]$. We observe a transition from normal to oblique dielectric rolls (a Lifshitz point) for the first time. We pay special attention to extreme low frequency driving and to the transition to dc voltage, where we investigate in detail the crossover from dielectric convection to flexodomains, i.e., the transition from a longitudinal to a transversal pattern.

The paper is organized as follows. The experimental setup is described in Sec. II, while the measurement technique is explained in Sec. III. In Sec. IV, the experimental results for the threshold characteristics of the observed patterns are discussed and compared with a fit to a theoretical model of our system. Finally, a conclusion is given in Sec. V.

\section{EXPERIMENTAL SETUP}

The liquid crystal "Merck N4" (also called "phase 4" [37]) is filled into a commercially available cell [38]. It consists of two parallel glass plates, which are separated by spacers and which have an indium tin oxide (ITO) layer on the inside. The ITO surfaces of the electrodes are coated with a polymer and rubbed in one direction in order to produce the planar alignment of the liquid crystal: When the cell is filled with a nematic material, the director is aligned along the rubbing direction in the layer plane. This direction defines the $x$ axis (compare Fig. 1), with the $y$ axis being perpendicular to $x$ in the layer plane. The thickness $d$ of the cell 
in the $z$ direction is determined by the spacers between the glass plates. We measure $d=11.4 \mu \mathrm{m}$ for the empty cell.

Either a dc voltage $U_{\mathrm{dc}}$ or a sinusoidal ac voltage $U_{\mathrm{ac}}(t)$ $=U \sin (2 \pi f t)$ is applied across the electrodes by means of a wave-form generator (Hewlett Packard 33120A) and an amplifier. The voltage is measured with a digital multimeter (Agilent 34410A). The samples are illuminated by a light emitting diode and the patterns are observed under a polarizing microscope (Olympus BX41) using the shadowgraph technique [17]. The light is polarized along the preferred direction, i.e., along $x$. Pictures of the $x-y$ plane are recorded with a CCD camera (Lumenera Lu135). The images have a spatial resolution of $1392 \times 1040$ pixels (physical size 316 $\times 236 \mu \mathrm{m}^{2}$ ) and a maximal contrast of 256 gray scales. Examples are shown in Fig. 1 (note that only quadratic subsections of the full images are displayed here). The measurements are performed at a temperature of $20^{\circ} \mathrm{C}$, which is stabilized to $\pm 5 \mathrm{mK}$ on top of the glass plates. The temperature increase caused by the ohmic heating inside the liquid crystal is estimated to be of the order of $1 \mathrm{mK}$. The complete setup is computer controlled.

\section{MEASUREMENT TECHNIQUE}

The formation of patterns is studied by starting from a homogeneous planar orientation (subcritical state) and then increasing the applied voltage $U$ in small steps for a fixed frequency $f$. Before each measurement, the system is given enough time to relax into the new state.

Dielectric convection rolls are often observed using a stroboscopic illumination: The light source flashes with the frequency $f$ of the driving ac voltage and is lit for only a short time $\tau \ll \frac{1}{f}$ and phase shifted by $\varphi$ with respect to the driving voltage [21]. According to the theory, the $z$ component of the director $\hat{n}$ oscillates in leading order as $n_{z}$ $=n_{0} \cos \left[2 \pi\left(f t-\varphi_{0}\right)\right]$. Thus, the visible convection pattern is time dependent with the same frequency as the driving voltage, so that the contrast of the shadowgraph image is largest at $\varphi=\varphi_{0}$ and at $\varphi=\varphi_{0}+\frac{1}{2}$. Note that we measure $\varphi$ in units of $2 \pi$. To detect the director oscillations, the measurements need to be taken as a function of $\varphi$. In order to increase the signal-to-noise ratio, many such measurements may be averaged.

At very low frequencies, a slightly different technique is used in order to save time: Instead of periodically flashing the light source, we use a permanent illumination of the liquid crystal, together with a very short exposure time of the CCD camera, which is phase shifted with respect to the driving voltage. In this way, a snapshot for the required phase shift is made and the measurements for varying $\varphi$ can in principle all be done during one period. In these experiments, we always use a constant exposure time of $1 \mathrm{~ms}$ and divide each period into 64 intervals, i.e., we take 64 snapshots and thus have measurements for 64 different phase shifts $\varphi$ during one period. This number is a compromise between camera speed, exposure time, and appropriate temporal resolution of the time-dependent patterns. For very low frequencies, these 64 snapshots are all taken during the same period of the driving voltage, while for frequencies larger

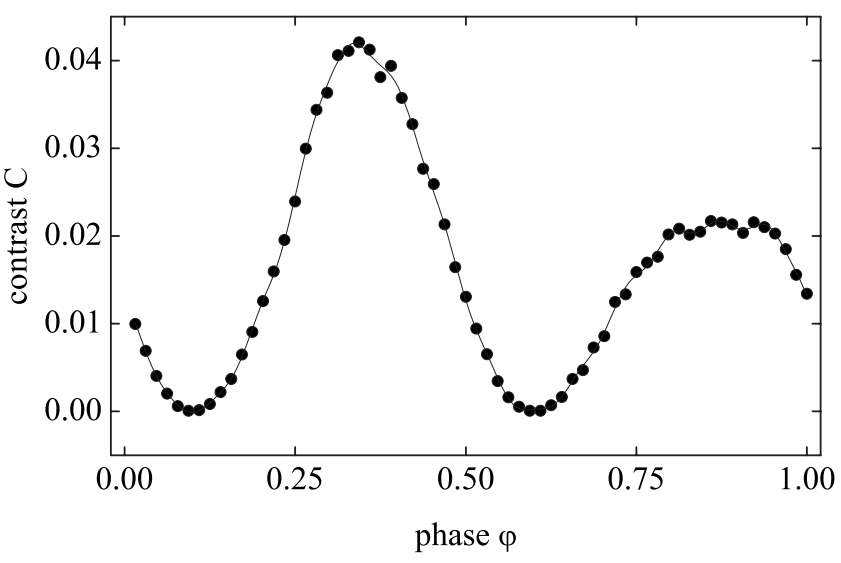

FIG. 2. Phase-dependent contrast along the $x$ axis shown for one period of the driving ac voltage at $f=4 \mathrm{~Hz}$ and $U=13.05 \mathrm{~V}$. The maximal contrast is reached at $\varphi_{0}=0.36$. This measurement corresponds to the oblique dielectric convection rolls shown in Fig. 1(b). The line is a guide to the eye.

than $f=0.8 \mathrm{~Hz}$, the 64 snapshots are taken in consecutive periods.

In order to obtain a measure for the amplitude of the respective patterns, we use the normalized intensity of a horizontal or vertical line from the shadowgraph images. In the case of electroconvection [see Figs. 1(b) and 1(c)], a line in the $x$ direction and in the case of flexodomains [see Fig. 1(a)], a line in the $y$ direction is taken. This line is divided pixelwise by a background line taken at a subcritical voltage, before a discrete Fourier series of the line is computed. We calculate the contrast $C$ of such a line as

$$
C=\sum_{k=N-2}^{N+2}\left(a_{k}^{2}+b_{k}^{2}\right),
$$

where $a_{k}$ and $b_{k}$ are the respective Fourier coefficients and $N$ is the mode corresponding to the critical wave number. To diminish leakage effects, the sum is taken over five modes. $C$ is a measure for the intensity of the critical mode [17].

Since the director oscillates with the driving frequency, $C$ is a (periodic) function of the phase shift $\varphi$. An example for oblique dielectric convection rolls is shown in Fig. 2 for a supercritical voltage, which corresponds to Fig. 1(b). The solid line in Fig. 2 represents a fit of a Fourier series to the data. For this and similar plots shown later in this paper, the first 15 coefficients were sufficient to reduce the maximal relative deviation between the data and the fitted curve to below $10 \%$. The contrast shows a periodic variation with a maximal contrast at $\varphi_{0}=0.36$. The fact that the local maximum at $\varphi=0.86$ is smaller (see also Figs. 8 and 10 below) is not yet understood in detail, but is most likely a manifestation of flexoelectric effects, which break the plus-minus symmetry with respect to the driving voltage. It is worth mentioning that no hint of a strong dependence of this feature on the distance from threshold could be found, although we did not attempt a systematic investigation.

In order to obtain the threshold voltage, the contrast at $\varphi_{0}$ is evaluated as a function of the applied voltage. $\varphi_{0}$ is chosen from that measurement, where the first supercritical pattern 


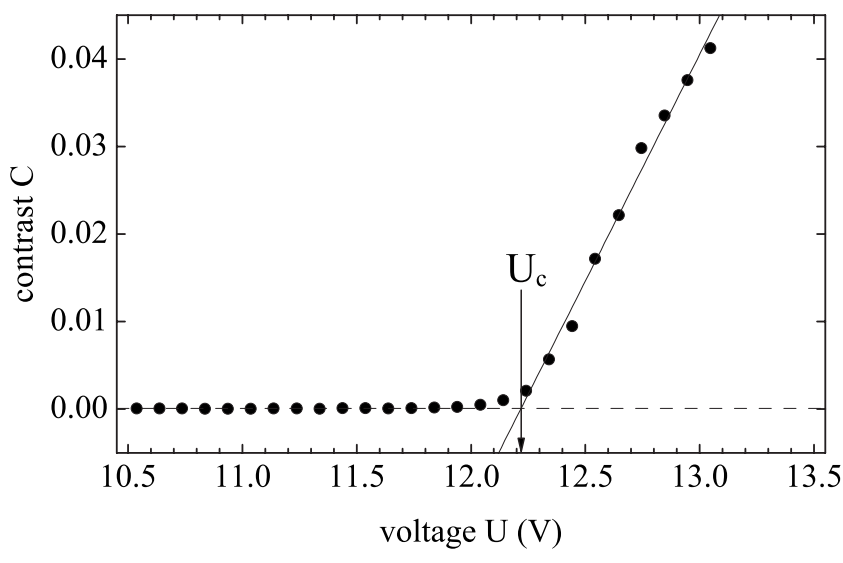

FIG. 3. Onset of oblique dielectric convection rolls for $f$ $=4 \mathrm{~Hz}$ and $\varphi=0.36$ for maximal contrast. The dashed and the solid lines are linear fits. Their intersection yields the threshold voltage $U_{c}=12.22 \mathrm{~V}$.

is detected, and it hardly varies with increasing voltage. An example for oblique dielectric convection rolls at $f=4 \mathrm{~Hz}$ is shown in Fig. 3. Guided by the model for the onset of the instability, we use a constant contrast for subcritical voltages and a linearly increasing contrast for supercritical voltages. A fit of this dependence to the measurement points is shown by the dashed (subcritical) and solid lines (supercritical) in Fig. 3 . The threshold voltage $U_{c}=12.22 \mathrm{~V}$ is determined by the intersection of the two lines. Note that for ac voltages, $U$ denotes the voltage amplitude and not the effective voltage.

\section{RESULTS AND DISCUSSION}

For a small cell thickness and a low electric conductivity of the liquid crystal, the conductive regime of electroconvection may not occur at all, which is the case in our sample. Moreover, a transition from dielectric convection to flexoelectric domains is observed when decreasing the frequency. The frequency dependence of the pattern formation is investigated by starting at a frequency of $f=200 \mathrm{~Hz}$ and then decreasing the frequency in successively smaller steps until the dc voltage is reached. For a better overview, the measurements are divided into two regions, which are discussed separately: A high-frequency range for $f>1 \mathrm{~Hz}$, and a lowfrequency range for $f<1 \mathrm{~Hz}$. The transition from oblique dielectric rolls to flexodomains in the low-frequency range is also discussed independently.

\section{A. High-frequency range}

For frequencies larger than $1 \mathrm{~Hz}$, only dielectric rolls are observed at onset. Their linear characteristics are shown in Fig. 4.

The critical voltage $U_{c}$ is indicated in Fig. 4(a). For frequencies $f \geqslant 15 \mathrm{~Hz}$, the phase $\varphi_{0}=0.38$ has been used for determining the threshold voltage. For lower frequencies, the maximum of the contrast occurs for smaller phase shifts: $\varphi_{0}=0.36$ for $f=4 \mathrm{~Hz}$ (compare Figs. 2 and 3) and $\varphi_{0}=0.34$ for $f=2 \mathrm{~Hz}$. The contrast as a function of the phase still shows a curve similar to the one depicted in Fig. 2. The

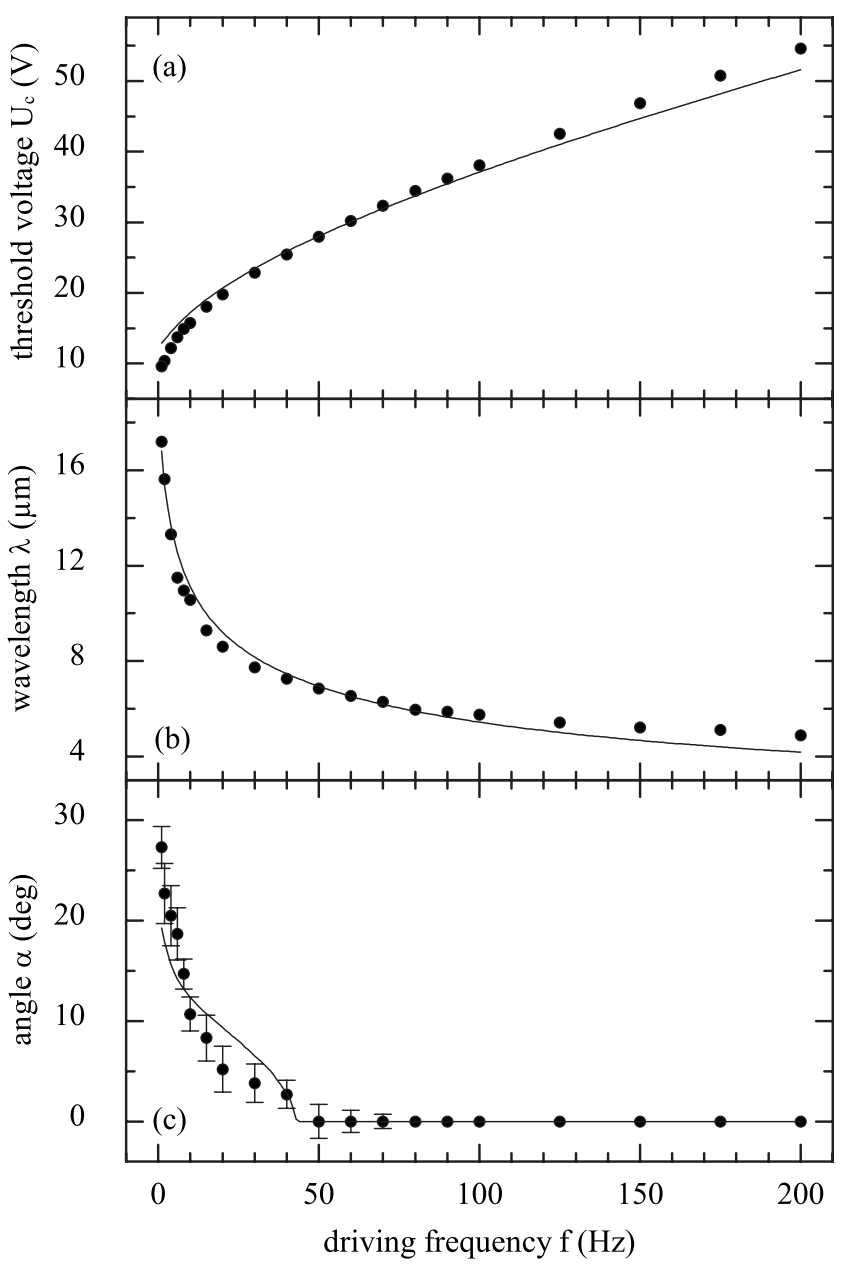

FIG. 4. Shown as functions of the driving frequency for $f$ $>1 \mathrm{~Hz}$ (high-frequency range) are (a) the threshold voltage $U_{c}$, (b) the wavelength $\lambda$, and (c) the angle of obliqueness $\alpha$. The lines represent the theoretical results.

threshold voltage increases for increasing frequencies, as predicted by the theory for dielectric convection rolls $[7,8]$.

The wavelength $\lambda$ of the pattern is shown in Fig. 4(b). It is determined from a two-dimensional Fourier transform of a shadowgraph image obtained at $2 \%$ above the threshold voltage. The monotonic decrease with increasing frequency is characteristic for dielectric rolls $[7,8]$.

For frequencies smaller than $50 \mathrm{~Hz}$, oblique dielectric rolls are observed at the onset of convection. Their angle of obliqueness is also determined from the two-dimensional Fourier transform of a shadowgraph image. This angle is shown as a function of the frequency in Fig. 4(c). The error bars represent the "full width half-maximum" value of the spectral peak. These measurements indicate a Lifshitz point near $50 \mathrm{~Hz}$, with normal rolls being observed for larger frequencies. This is in fact the first experimental observation of oblique dielectric rolls at onset.

The lines in Fig. 4 are the results of a theoretical description of the dielectric regime of electroconvection, which is based on an extended standard model including flexoelectricity [28]. They have been obtained from a linear stability analysis of the underlying nematohydrodynamic equations. An applied ac voltage of the form $U \sin (2 \pi f t)$, strong planar 
TABLE I. Material parameters of $N 4$ at a temperature of $T$ $=20^{\circ} \mathrm{C}$.

\begin{tabular}{lccc}
\hline \hline Parameter & Unit & Value & Reference \\
\hline$\alpha_{1}$ & & -10.1 & $($ Fit $)$ \\
$\alpha_{2}$ & & -204.3 & {$[29,30]$} \\
$\alpha_{3}$ & 4.8 & {$[29,30]$} \\
$\alpha_{4}$ & $10^{-3} \mathrm{~Pa} \mathrm{~s}$ & 103.0 & {$[29,30]$} \\
$\alpha_{5}$ & & 146.7 & {$[29,30]$} \\
$\alpha_{6}$ & & -52.8 & {$[29,30]$} \\
\hline$k_{11}$ & & 9.1 & $($ Fit $)$ \\
$k_{22}$ & $10^{-12} \mathrm{~N}$ & 5.9 & $($ Fit $)$ \\
$k_{33}$ & & 14.1 & $($ Fit $)$ \\
\hline$\sigma_{\perp}$ & $10^{-10}(\Omega \mathrm{m})^{-1}$ & 5.0 & (Fit) \\
\hline$\sigma_{a} / \sigma_{\perp}$ & & 0.28 & (Fit) \\
$\epsilon_{\perp}$ & 5.0 & (Fit) \\
$\epsilon_{a}$ & & -0.1 & (Fit) \\
\hline$e_{1}$ & & -18.1 & (Fit) \\
$e_{3}$ & $10^{-12} \mathrm{C} / \mathrm{m}$ & -13.4 & (Fit) \\
\hline \hline
\end{tabular}

anchoring for the director and realistic no-slip boundary conditions for the velocity have been assumed. For a quantitative comparison with the experimental data at the onset of electroconvection, the thickness $d=11.4 \mu \mathrm{m}$ of the nematic layer was taken from the measurement of the empty cell and the mass density was taken to be $\rho=10^{3} \mathrm{~kg} / \mathrm{m}^{3}$. The other material parameters used for the lines in Fig. 4 are given in Table I.

Considering the Parodi relation, Table I leaves us with 14 independent material parameters, which in principal can all be adjusted for a fit to the measurements shown in Fig. 4. It turns out, however, that a perfect match (within the experimental errors) could not be obtained, even when all parameters are used as fit parameters. Moreover, using too many fit parameters seems prohibitive, because the uncertainty of each single material constant obtained from the fit will increase with the number of free parameters. In seeking a suitable compromise, we decided to take the available viscosity coefficients from the literature and fit the remaining parameters. The five viscosity coefficients $\alpha_{2}, \ldots, \alpha_{6}$ have thus been obtained from measurements of the Miesowicz shear viscosities $\eta_{1}, \eta_{2}, \eta_{3}$ [29] and the rotational viscosity $\gamma_{1}$ [30], taking into account the Parodi relation. A value for the viscosity coefficient $\alpha_{1}$ is not available from the literature and has thus been fitted. The other fit parameters are the elastic constants $k_{11}, k_{22}$, and $k_{33}$, the electric conductivity $\sigma_{\perp}$ and the anisotropy of conductivity $\sigma_{a}=\sigma_{\|}-\sigma_{\perp}$, the dielectric permittivity $\epsilon_{\perp}$, and the dielectric anisotropy $\epsilon_{a}=\epsilon_{\|}$ $-\epsilon_{\perp}$, and the flexoelectric coefficients $e_{1}$ and $e_{3}$. In addition to the experimental data shown in Fig. 4 , the measured critical voltage and wavelength of the flexoelectric domains at dc voltage have also been used for the fitting procedure. Note that the latter two depend only on four material parameters, namely $k_{11}, k_{22}, \epsilon_{a}$ and the combination of the flexoelectric coefficients $e_{1}-e_{3}$. The onset characteristics of the electroconvection depend in addition on $\alpha_{i}, k_{33}, \epsilon_{\perp}, \sigma_{\perp}, \sigma_{a}$, and $e_{1}+e_{3}$. Our parameter study has shown, that the onset characteristics of the dielectric electroconvection are mostly sensitive to the values of $e_{1}, e_{3}, \frac{\sigma_{a}}{\sigma_{\perp}}$, and $\frac{\epsilon_{a}}{\epsilon_{\perp}}$. In addition, oblique dielectric rolls were not found in the absence of flexoelectricity, i.e., for $e_{1}=e_{3}=0$.

The material parameters resulting from the fitting procedure (see Table I) are quite realistic. In particular, the elastic constant $k_{33}$ is consistent with the measurements at $T$ $=30{ }^{\circ} \mathrm{C}$ and $T=25^{\circ} \mathrm{C}$ reported in Ref. [31]. The flexoelectric coefficients are in the range of known values for the nematic liquid crystal MBBA, where the data obtained by different methods range from $-1 \mathrm{pC} / \mathrm{m}$ to $-20 \mathrm{pC} / \mathrm{m}$ for $e_{1}$ and from $-4 \mathrm{pC} / \mathrm{m}$ to $-35 \mathrm{pC} / \mathrm{m}$ for $e_{3}[13,32]$. The values for $\epsilon_{\perp}$ and $\epsilon_{a}$ are in good agreement with our own measurements, using the method discussed in Ref. [33]. The calculated critical dc voltage for the flexodomains $U_{c}^{\mathrm{dc}}=13.9 \mathrm{~V}$ and the critical wavelength $\lambda_{c}^{\mathrm{dc}}=15.1 \mu \mathrm{m}$ are in a good agreement with the experimental data (see next section).

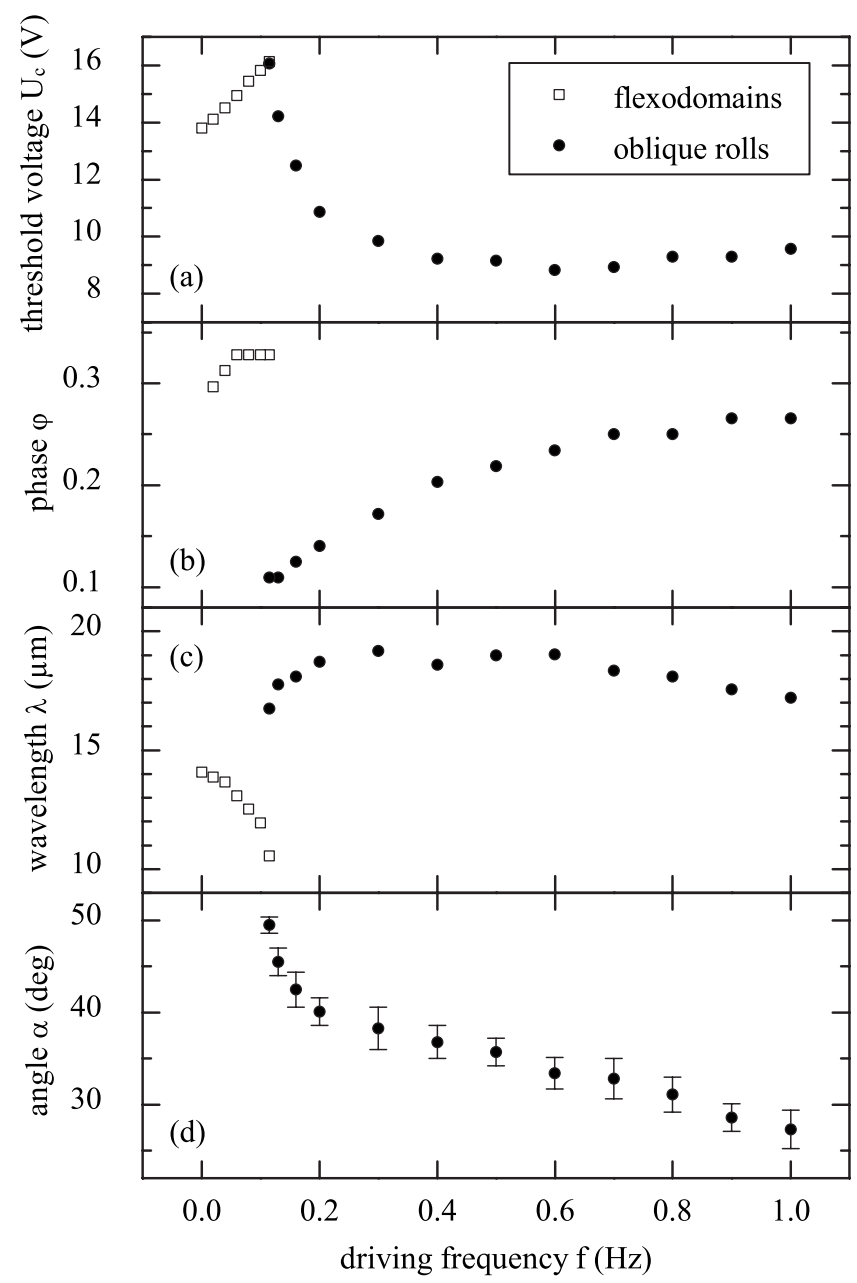

FIG. 5. Shown as functions of the driving frequency for $f$ $<1 \mathrm{~Hz}$ (low-frequency range) are (a) the threshold voltage $U_{c}$, (b) the phase $\varphi$ for maximal contrast, (c) the wavelength $\lambda$, and (d) the angle of obliqueness $\alpha$. 


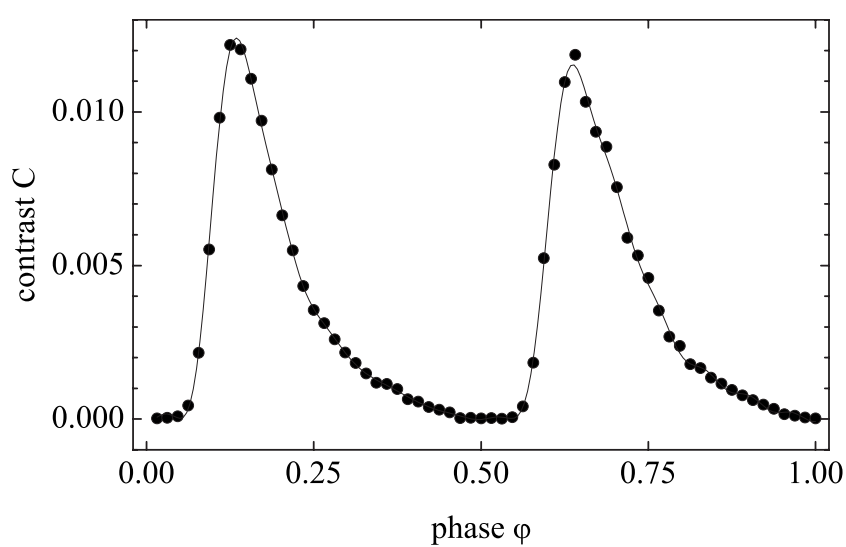

FIG. 6. Phase-dependent contrast along the $x$ axis shown for one period of the driving ac voltage at $f=200 \mathrm{mHz}$ and $U=12.05 \mathrm{~V}$. The maximal contrast is reached at $\varphi_{0}=0.14$. This measurement corresponds to oblique dielectric convection rolls. The line is a guide to the eye.

\section{B. Low-frequency range}

For frequencies smaller than $1 \mathrm{~Hz}$, the onset characteristics analogous to Fig. 4 are shown in Fig. 5. In this range, a transition from dielectric rolls to flexodomains occurs when decreasing the frequency.

The phase for which the maximal contrast is observed in the case of dielectric rolls shifts to lower values for decreasing frequencies [see Fig. 5(b)]. Between $0.6 \mathrm{~Hz}$ and $1 \mathrm{~Hz}$, it is around $\varphi_{0}=0.25$ which corresponds to the maximum of the applied ac voltage, before it decreases further to its minimal value of $\varphi_{0}=0.11$ at $f_{t}=115 \mathrm{mHz}$.

An example for the dependency of the contrast on the phase shift is shown in Fig. 6 for $f=200 \mathrm{mHz}$, where we find $\varphi_{0}=0.14$. The contrast increases very quickly and then slowly decreases again.

For determining the onset, the same method as above has been used: A constant contrast for subcritical voltages and a linear increase above threshold, which is shown in Fig. 7. For small frequencies, the threshold voltage decreases from

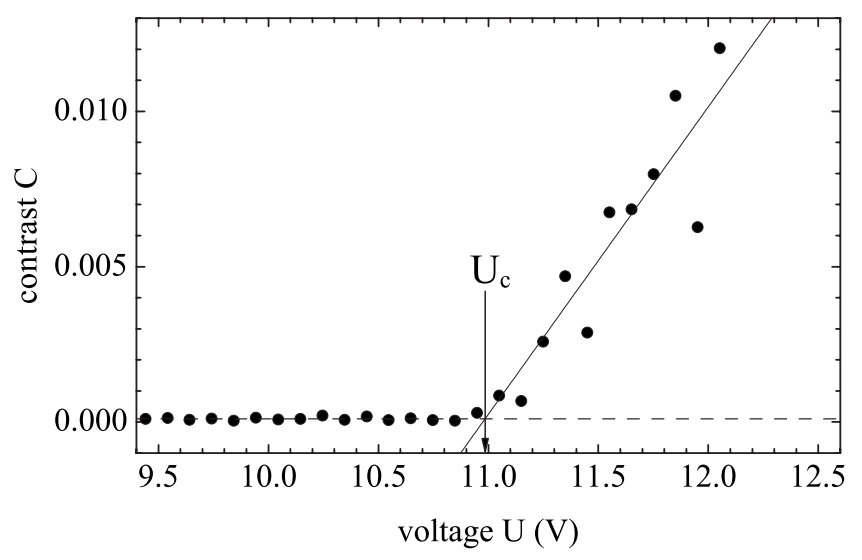

FIG. 7. Onset of oblique dielectric convection rolls for $f$ $=200 \mathrm{mHz}$ and $\varphi=0.14$ for maximal contrast. The dashed and the solid lines are linear fits. Their intersection yields the threshold voltage $U_{c}=10.98 \mathrm{~V}$.

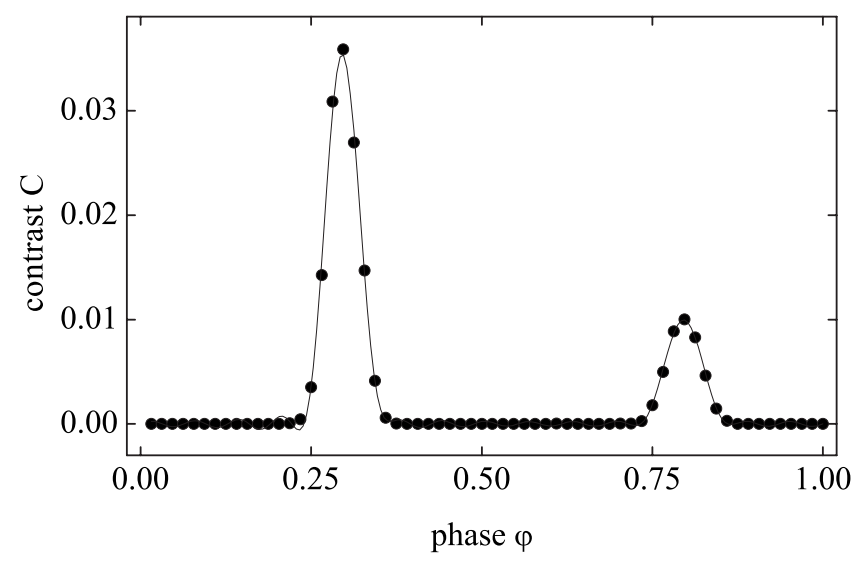

FIG. 8. Phase-dependent contrast along the $y$ axis shown for one period of the driving ac voltage at $f=20 \mathrm{mHz}$ and $U=15.06 \mathrm{~V}$. The maximal contrast is reached at $\varphi_{0}=0.30$. This measurement corresponds to flexoelectric domains. The line is a guide to the eye.

an apparently divergent behaviour at $f \rightarrow 0 \mathrm{~Hz}$. $U_{c}$ has a minimum at $f_{m} \approx 600 \mathrm{mHz}$ [see Fig. 5(a)]. This minimum is expected to be determined by $\frac{1}{\tau_{d}}$ [34], where $\tau_{d}=\frac{\gamma_{1} d^{2}}{k_{11}}$ is the director relaxation time [7], which in our case is $\tau_{d} \approx 3 \mathrm{~s}$.

The linear stability analysis performed with the material parameters from Table I does not reveal the tendency of the threshold voltage to increase with decreasing frequency, at least not down to $f=0.25 \mathrm{~Hz}$. These low-frequency calculations become highly time consuming due to the less efficient temporal Fourier expansion of the linear modes. The dynamics, in particular of the director, become more and more spiky, as can also be seen in the experiments, e.g., in Fig. 6. The increase of the critical voltage of the oblique dielectric rolls close to the transition to the flexodomains could be related to nonohmic conductivity effects, which are not included in our model.

The threshold voltage has a local maximum at the transition frequency $f_{t}=115 \mathrm{mHz}$. This is the lowest frequency for which dielectric rolls are observed. For frequencies below $f_{t}$, flexodomains appear instead [compare Fig. 1(a)], because their threshold is lower than the one for dielectric convection rolls. The critical voltage for the ac flexodomains shows a linear dependence on the frequency: $U_{c}=13.67 \mathrm{~V}$ $+21.57 \mathrm{~V} / \mathrm{Hz} f$ [see Fig. 5(a)]. We find for the critical dc voltage $U_{c}^{\mathrm{dc}}=13.81 \mathrm{~V} \approx U_{c}(f \rightarrow 0 \mathrm{~Hz})$, which is in agreement with the statement by Barnik et al. [24] that these voltages should match. This is still remarkable because for a deterministic calculation (without noise), a factor of $\sqrt{2}$ between the two values is expected.

For such low frequencies, the temporal behavior of the pattern changes dramatically. In Fig. 8, the contrast is shown for one period of the driving ac voltage at $f=20 \mathrm{mHz}$ : Most of the time no pattern is observed, and only for a short duration after the maximal voltage was applied to the cell, flexodomains are visible. Here, the period is obviously longer than the decay time of the domain structures. The curvature of the contrast seems to be symmetric around the maximum. The contrast difference between the two maxima is most likely due to flexoelectric effects. The phase for maximal contrast is $\varphi_{0}=0.30$ for $f=20 \mathrm{mHz}$ and increases for increas- 


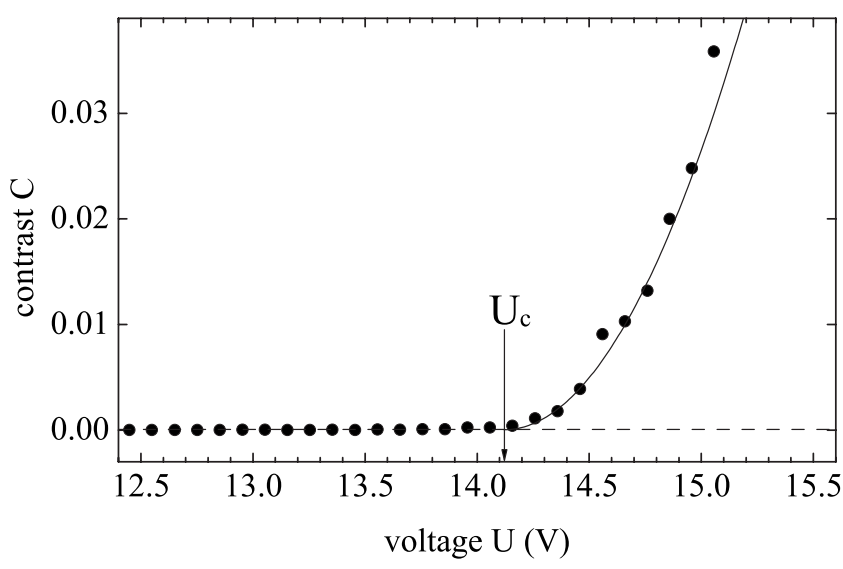

FIG. 9. Onset of flexoelectric domains for $f=20 \mathrm{mHz}$ and $\varphi$ $=0.30$ for maximal contrast. The dashed line is a linear fit, while the solid line represents a parabola. Their intersection yields the threshold voltage $U_{c}=14.12 \mathrm{~V}$.

ing frequencies to $\varphi_{0}=0.33$ for $f_{t}=115 \mathrm{mHz}$ [see Fig. 5(b)].

For determining the onset, we must take into accout the different optical behavior of the flexodomains as compared to electroconvection. For flexodomains, the director field is constant along the $x$ direction, while it varies harmonically along $y$. This yields a quadratic shadowgraph effect which is due to the bending of light inside a medium with varying refractive index, in contrast to the linear effect for electroconvection, which is due to light focusing outside the liquid crystal layer. Therefore, the contrast $C$ as a function of the driving voltage should show a quadratic dependence at threshold. We have obtained the threshold by fitting a straight line to the square root of the contrast, but in order to be consistent with Figs. 3 and 7, we again show $C$ as a function of the voltage in Fig. 9. The dependence above threshold is now represented by a parabola, while a constant contrast is fitted below threshold.

The wavelength $\lambda$ of the oblique dielectric rolls shows a slight dependence on the frequency [see Fig. 5(c)]. For decreasing frequencies, it first increases up to a maximum of $\lambda \approx 19 \mu \mathrm{m}$ before decreasing again. The maximal diameter of one convection roll is thus of the order of the cell thickness $(d=11.4 \mu \mathrm{m})$. The angle of obliqueness still increases for small frequencies from about $27^{\circ}$ for $f=1 \mathrm{~Hz}$ to its maximum of about $50^{\circ}$ for $f_{t}=115 \mathrm{mHz}$ [see Fig. 5(d)].

For flexodomains, the wavelength increases from about $11 \mu \mathrm{m}$ for $f_{t}=115 \mathrm{mHz}$ to about $14 \mu \mathrm{m}$ for $f=20 \mathrm{mHz}$ [see Fig. 5(c)]. The value obtained when a dc voltage is applied matches the wavelength obtained for ac voltages very well. No obliqueness was observed for the flexodomains.

\section{Transition between dielectric convection and flexoelectric domains}

The transition frequency $f_{t}=115 \mathrm{mHz}$ is the lowest frequency for which dielectric convection rolls were observed. If the applied ac voltage is increased slightly above the threshold for dielectric rolls, flexodomains arise and both patterns can be observed within one period of the driving voltage. This behavior can be illustrated by studying the de-

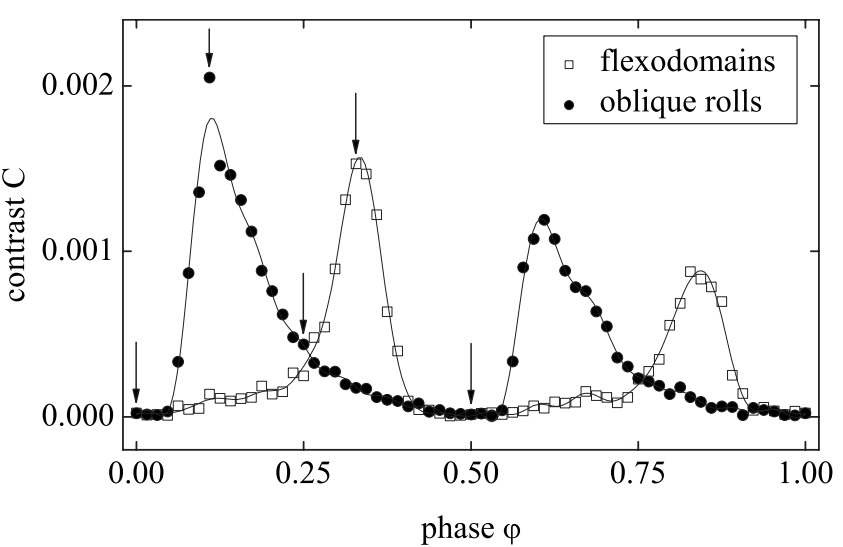

FIG. 10. Phase-dependent contrast along the $y$ axis shown for one period of the driving ac voltage at $f=115 \mathrm{mHz}$ and $U$ $=16.26 \mathrm{~V}$. The first and the third peak correspond to oblique dielectric convection rolls (here also shown along the $y$ axis), while the second and the fourth peak correspond to flexoelectric domains. Arrows mark the points in time shown in Fig. 11. The lines are guides to the eye.

pendence of the contrast on the phase shift, which is shown in Fig. 10. When varying the phase, four distinct maxima can be seen, with the first and the third corresponding to dielectric convection rolls (indicated by the filled circles) and the second and the fourth to flexodomains (indicated by the open squares). The two patterns have different wave numbers, which are constant during the cycle. The points marked by the arrows correspond to the snapshots shown in Fig. 11. Again we think, that the contrast differences between the first and the third peak and between the second and the fourth peak are due do flexoelectric effects.

At the beginning of the period $(\varphi=0.00)$, no pattern is observed. Oblique dielectric rolls emerge with a maximum of the contrast at $\varphi=0.11$. They decay again, but just before the initial noise level is reached flexodomains appear at $\varphi$ $=0.25$. They have a maximal contrast at $\varphi=0.33$, after which they decay again. After one-half of the period $(\varphi=0.50)$, the almost undisturbed ground state is reached and the transition from electroconvection to flexodomains repeats itself periodically in the same way.

Note that this transition is different from the crossover between flexoelectric domains and conductive electroconvection rolls observed in hybrid aligned nematic MBBA under dc voltage [35]. There, the electroconvection is developed well above the onset of the flexodomains on the background of a highly distorted director orientation.

\section{CONCLUSION}

In this paper, the pattern formation in an anisotropic system was studied. The starting point of the experiment was the observation of two periodic striped patterns perpendicular to each other in the shadowgraph image of the nematic liquid crystal "Merck N4." For our investigations, a liquid crystal layer with a thickness of $d=11.4 \mu \mathrm{m}$ was used. In this sample, no conductive regime of electroconvection was observed. When decreasing the frequency of the driving ac 


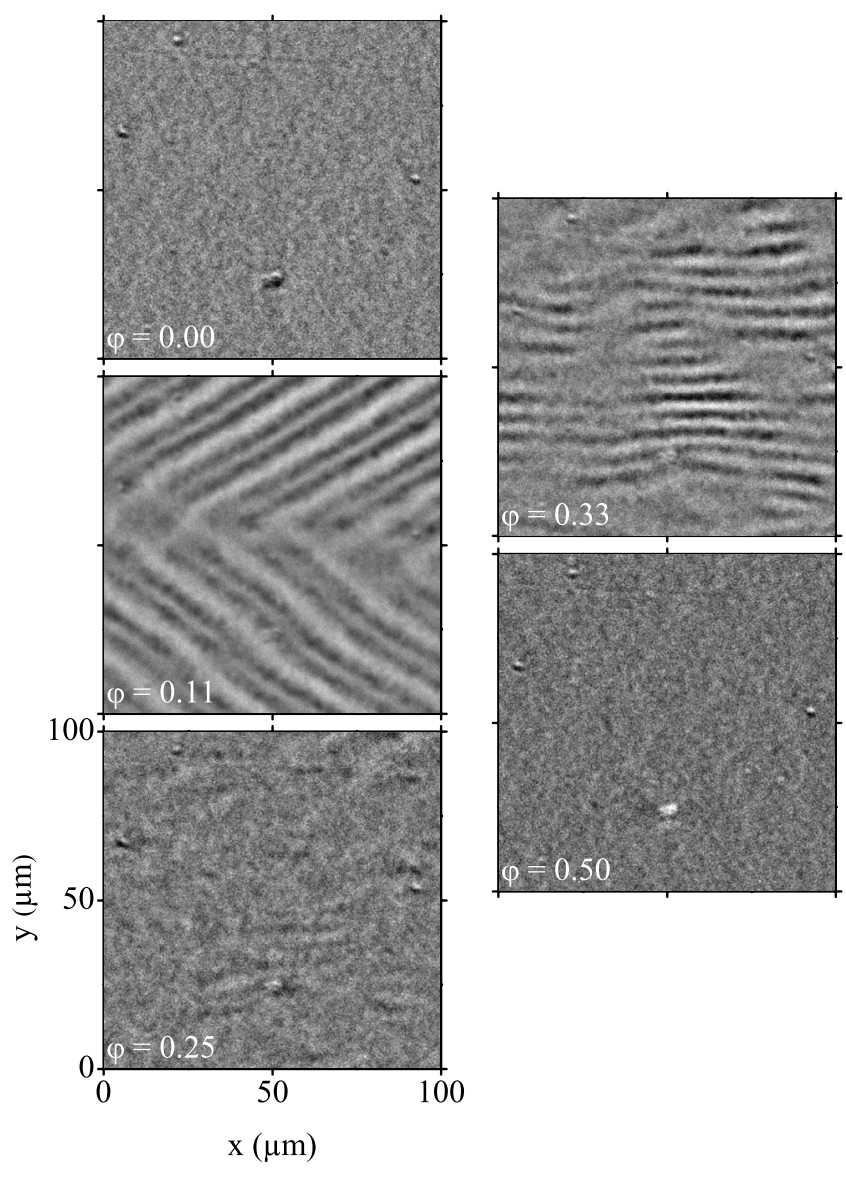

FIG. 11. Time series of snapshots during one half-period of the driving frequency at $f=115 \mathrm{mHz}$ and $U=16.26 \mathrm{~V}$ (compare Fig. 10).

voltage, the longitudinal pattern of the normal dielectric convection rolls undergoes a transition to the transversal pattern of the flexoelectric domains via oblique dielectric convection rolls.

Oblique dielectric convection at onset has not been reported in the literature so far, neither theoretically nor experimentally. We find a transition from normal to oblique rolls, i.e., a Lifshitz point, at a frequency of $f_{\mathrm{L}} \approx 50 \mathrm{~Hz}$. For decreasing frequency, the angle of obliqueness increases from $0^{\circ}$ to about $50^{\circ}$ for $f_{t}=115 \mathrm{mHz}$, the lowest frequency for which dielectric convection was observed in our sample. The threshold voltage and the critical wavelength of the convection patterns have been measured. It turns out, that the critical voltage has a minimum value for the frequency $f_{m}$ $\approx 600 \mathrm{mHz}$.

Using the extended electroconvection model including flexoelectricity, we were able to describe quantitatively the transition from normal to oblique dielectric rolls by a reason- able choice of material parameters. Without the flexoelectric effect, oblique dielectric rolls are not found in the theory. It is expected that combining flexoelectricity with the nonohmic conductivity, e.g., the weak electrolyte model [36], could give an explanation for the unusual divergentlike behavior of the critical voltage of the dielectric electroconvection at low frequency.

The transition from longitudinal flexoelectric domains to dielectric convection rolls presented here is an example of a codimension-two bifurcation. When considering primary instabilites in liquid crystals under an applied electric field, it thus appears comparable to three other bifurcations of this type. The first one is the crossover between the Fréedericksz and the Carr-Helfrich instability, a competition between a zero wave number and a pattern forming instability. The second one is the crossover between conductive and dielectric convection rolls, a competition between dissipative structures with different time symmetries. The third example is a Lifshitz point, i.e., the transition from normal to oblique rolls, which is a supercritical bifurcation between two modes of the Carr-Helfrich type. The transition scenario that we have presented here differs from these three scenarios: It is a crossover between two pattern forming (i.e., finite wave number) instabilities, one being of dissipative nature, while the other one is an equilibrium instability not involving any flow.

An important result of the paper is, that a transition frequency $f_{t}=115 \mathrm{mHz}$ has been found, at which both oblique dielectric rolls and flexodomains can be observed in turn, depending on the phase of the driving ac voltage. The reason for this is, that the maximal contrast for each pattern occurs at a different phase, and that for not too high voltages, the two patterns do not seem to influence each other, due to the different nature of the instabilities. The convection rolls arise from the homogeneous planar basic state and decay almost entirely before flexodomains develop. This sequence is repeated periodically in time.

Whether this mixed mode arises as a primary or secondary instability could not be resolved within the measurements presented here and remains a challenge for further experimental investigations. Moreover, a nonlinear theory capable of describing such a transition scenario is not yet available.

\section{ACKNOWLEDGMENTS}

The special trigger for the stroboscopic detection of the shadowgraph images was designed by Achim Sack. It is also a pleasure to thank Werner Pesch for clarifying discussions. Financial support by the Hungarian Research Grant No. OTKA-K61075 and the Deutsche Forschungsgemeinschaft Grant No. SFB 481 are gratefully acknowledged. 
[1] M. C. Cross and P. C. Hohenberg, Rev. Mod. Phys. 65, 851 (1993).

[2] F. H. Busse, Rep. Prog. Phys. 41, 1929 (1978).

[3] R. P. Behringer, Rev. Mod. Phys. 57, 657 (1985).

[4] J. W. Deardorff, Phys. Fluids 8, 1027 (1965).

[5] R. E. Kelly, Adv. Appl. Mech. 31, 35 (1994).

[6] W. W. Mullins and R. F. Sekerka, J. Appl. Phys. 35, 444 (1964).

[7] L. Kramer and W. Pesch, in Pattern Formation in Liquid Crystals, edited by A. Buka and L. Kramer (Springer, New York, 1996), p. 221.

[8] L. Kramer and W. Pesch, in Physical Properties of Liquid Crystals: Nematics, edited by D. A. Dunmur, A. Fukuda, and G. R. Luckhurts (Inspec, London, 2001), p. 441.

[9] A. Buka, N. Éber, W. Pesch, and L. Kramer, in Self Assembly, Pattern Formation and Growth Phenomena in Nano-Systems, edited by A. A. Golovin and A. A. Nepomnyaschy (Kluwer Academic, New York, 2006), p. 55.

[10] T. Tóth-Katona, A. Cauquil-Vergnes, N. Éber, and A. Buka, Phys. Rev. E 75, 066210 (2007).

[11] J. Heuer, R. Stannarius, M. G. Tamba, and W. Weissflog, Phys. Rev. E 77, 056206 (2008).

[12] P. G. de Gennes and J. Prost, The Physics of Liquid Crystals (Clarendon, Oxford, 1993).

[13] S. Chandrasekhar, Liquid Crystals (Cambridge University Press, Cambridge, 1994).

[14] A. Buka and L. Kramer, Pattern Formation in Liquid Crystals (Springer, New York, 1996).

[15] E. F. Carr, Mol. Cryst. Liq. Cryst. 7, 253 (1969).

[16] W. Helfrich, J. Chem. Phys. 51, 4092 (1969).

[17] S. Rasenat, G. Hartung, B. L. Winkler, and I. Rehberg, Exp. Fluids 7, 412 (1989).

[18] E. Bodenschatz, W. Zimmermann, and L. Kramer, J. Phys. (Paris) 49, 1875 (1988)

[19] W. Thom, W. Zimmermann, and L. Kramer, Liq. Cryst. 4, 309 (1989).

[20] S. Rasenat, V. Steinberg, and I. Rehberg, Phys. Rev. A 42, 5998 (1990).

[21] U. Schneider, M. de la Torre Juárez, W. Zimmermann, and I.
Rehberg, Phys. Rev. A 46, 1009 (1992).

[22] R. B. Meyer, Phys. Rev. Lett. 22, 918 (1969).

[23] Y. P. Bobylev and S. A. Pikin, Sov. Phys. JETP 45, 195 (1977).

[24] M. I. Barnik, L. M. Blinov, A. N. Trufanov, and B. A. Umanski, Sov. Phys. JETP 46, 1016 (1977).

[25] L. Kramer, E. Bodenschatz, W. Pesch, W. Thom, and W. Zimmermann, Liq. Cryst. 5, 699 (1989).

[26] N. V. Madhusudana and V. A. Raghunathan, Liq. Cryst. 5, 1789 (1989).

[27] W. Zimmermann, in Defects, Singularities and Patterns in Nematic Liquid Crystals, edited by J. M. Coron, J. Ghidaglia, and F. Helein, NATO Advanced Study Institute Series (Kluwer, Dordrecht, 1991).

[28] A. Krekhov, W. Pesch, N. Éber, T. Tóth-Katona, and A. Buka, Phys. Rev. E 77, 021705 (2008).

[29] H. Kneppe, F. Schneider, and N. K. Sharma, Ber. Bunsenges. Phys. Chem. 85, 784 (1981).

[30] H. Kneppe, F. Schneider, and N. K. Sharma, J. Chem. Phys. 77, 3203 (1982).

[31] D. Funfschilling, B. Sammuli, and M. Dennin, Phys. Rev. E 67, 016207 (2003).

[32] A. G. Petrov, in Physical Properties of Liquid Crystals: Nematics, edited by D. A. Dunmur, A. Fukuda, and G. R. Luckhurst (Inspec, London, 2001), p. 251.

[33] M. Müller, W. Schöpf, I. Rehberg, A. Timme, and G. Lattermann, Phys. Rev. E 76, 061701 (2007).

[34] T. Tóth-Katona, N. Éber, A. Buka, and A. Krekhov, Phys. Rev. E 78, 036306 (2008).

[35] V. A. Delev, A. P. Krekhov, and L. Kramer, Mol. Cryst. Liq. Cryst. Sci. Technol., Sect. A 366, 849 (2001).

[36] M. Treiber and L. Kramer, Mol. Cryst. Liq. Cryst. Sci. Technol., Sect. A 261, 311 (1995).

[37] Nematic phase 4, art.no. 10105; Merck KGaA, Frankfurter Str. 250, 64293 Darmstadt, Germany. N4 is a eutectic mixture of the two azoxy compounds $\mathrm{CH}_{3} \mathrm{O}-\mathrm{C}_{6} \mathrm{H}_{4}-$ $\mathrm{NO}=\mathrm{N}-\mathrm{C}_{6} \mathrm{H}_{4}-\mathrm{C}_{4} \mathrm{H}_{9}$ and $\mathrm{CH}_{3} \mathrm{O}-\mathrm{C}_{6} \mathrm{H}_{4}-\mathrm{N}=\mathrm{NO}-$ $\mathrm{C}_{6} \mathrm{H}_{4}-\mathrm{C}_{4} \mathrm{H}_{9}$.

[38] E.H.C. Co. Ltd., 1164 Hino, Hino-shi, Tokyo, Japan 191. 